\title{
Mathematical model of flow through the patent ductus arteriosus
}

\author{
Adriana Setchi · A. Jonathan Mestel . \\ Jennifer H. Siggers · Kim H. Parker . \\ Ming Wang Tan · Kangwen Wong
}

Received: date / Accepted: date

\begin{abstract}
The ductus arteriosus is one of several shunts in the cardiovascular system. It is a small vessel connecting the aortic arch and pulmonary artery that allows blood to bypass the pulmonary circulation. It is open during foetal development because the foetal lungs cannot function and oxygenation of the blood occurs by exchange with the maternal blood in the placenta. Normally it closes a few days after birth. However, in a small number of people closure does not occur, leading to a condition known as patent ductus arteriosus. In this paper our aim is to investigate the resulting cardiovascular effects. We develop a mathematical model of the haemodynamics in three different idealised geometries by assuming that the entry flow is irrotational and remains so in the core until at least the shunt position. We argue that separation or diffusion of vorticity into the core flow is delayed due to the high frequency associated with the pulsatile component of the flow profile. The analysis uses complex potential theory, Schwarz-Christoffel transformations, conformal mappings and Fourier series. The main results are based on the assumption that the flow in patients with patent ductus arteriosus is similar to the flow in healthy adults, and we apply this assumption using boundary conditions that are representative of physiological values in healthy adults. The model suggests that the pressures in the aorta and pulmonary artery are likely to equalise, that the shear stress increases near the edges of the shunt and that backflow of large volumes may occur from the pulmonary artery into the aorta due to the presence of the patent shunt.
\end{abstract}

Keywords Patent ductus arteriosus - Cardiovascular shunt $\cdot$ Potential flow $\cdot$ High Womersley number

A. Setchi, J. Siggers, K. Parker, M. W. Tan, K. Wong

Department of Bioengineering, Imperial College London, SW7 2AZ, UK

E-mail: asetchi@imperial.ac.uk,j.siggers@imperial.ac.uk,k.parker@imperial.ac.uk

J. Mestel

Department of Mathematics, Imperial College London, SW7 2AZ, UK

E-mail: j.mestel@imperial.ac.uk 

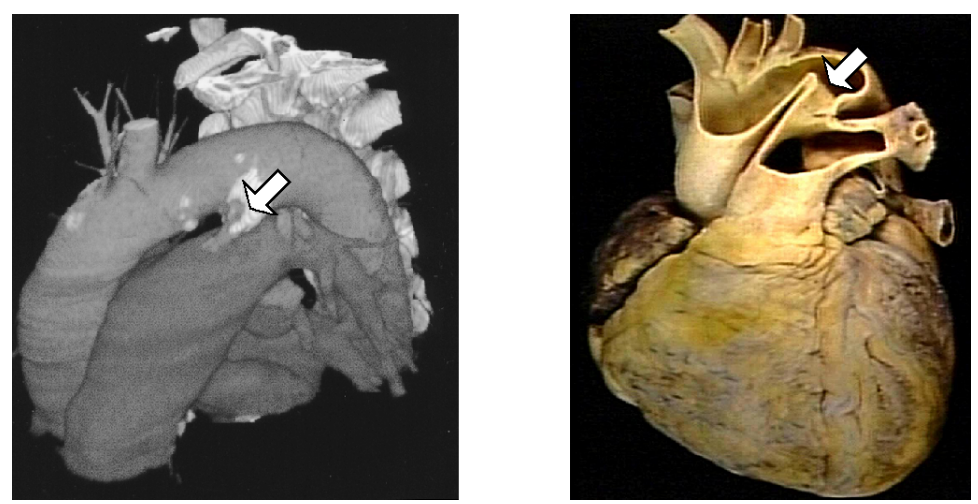

Fig. 1 A 3-D CT reconstruction (left) and cross-sectional view (middle) of patent ductus arteriosus geometries (marked by arrows), aorta (vessel above) and pulmonary artery (vessel below). Images after [1] and [2].

\section{Introduction}

The ductus arteriosus is a vascular connection between the pulmonary artery and the aortic arch in the developing foetus that ensures oxygen is efficiently transported around the foetal circulation. It protects the right ventricle from pumping against the high resistance in the fluid-filled lungs by shunting most of the flow from the right ventricle into the aorta. Usually, it is functionally closed within days after birth, and is completely sealed after three weeks. Failure for this to happen results in a condition called patent ductus arteriosus (PDA). Typical geometries of the PDA are shown in Figure 1 . The mortality rate if the PDA does not close and is left untreated is assessed to be $20 \%, 42 \%$ and $61 \%$ by ages 20,45 and 60 years respectively $[3,4]^{1}$. If left uncorrected, irregular transmission of blood between the two largest arteries in the body can lead to severe health complications such as heart failure.

Surprisingly, many sufferers of PDA appear asymptomatic, even in the presence of significantly large shunts. Such cases are medically fascinating as, paradoxically, the cardiovascular defect appears to have little qualitative effect on the circulation. For example, a 55-year-old marathon runner of excellent exercise capacity was found to have a shunt with large cross-section $0.0125 \times 0.008 \mathrm{~m}^{2}$ [5]. Other asymptomatic PDA patients experience healthy blood pressures in both the aorta and pulmonary artery despite significant flows between the two vessels [6-8]. Most PDA sufferers develop cardiomegaly or dilated blood vessels, pulmonary hypertension or a lower pressure difference between the two arteries. These observations suggest that the heart and lungs adapt in order to compensate for the defect. This hypothesis is also supported by measurements in symptomatic PDA patients $[9,10]$ and in foetal lambs $[11,12]$. Due to the severity of PDA and the complexities associated with correcting it surgically or non-invasively with an occluding stent, it is important to diagnose it early, to know the side effects and to predict its development. In particular, knowledge

1 These rates in healthy adults are $1 \%, 5 \%$ and $12 \%$ as estimated by insurance companies in 2012 
of the blood flow distribution and of the vascular pressures is crucial to a better understanding of the condition and its responses to stress and disease.

Two main theoretical approaches can be adopted to study the flow through a PDA. The full patient-specific problem can be tackled using a numerical simulation; alternatively, the problem can be idealised using simplified geometry and equations governing the mechanics in order to gain insight into the underlying mechanisms. In this paper we adopt the latter approach for three reasons. Firstly, the dominant mechanisms in the haemodynamics of asymptomatic PDA patients can be found from analysis of key non-dimensional quantities, which can guide future computational or experimental research. Secondly, due to difficulties in measurements, there are limited experimental data available on the shunt geometry and blood flow. Idealised analytical solutions can provide generic information about the effects of different, poorly characterised parameters which are difficult or impossible to obtain with numerical modelling. Thirdly, the anatomy varies significantly between different subjects, for example in the size, shape and location of the PDA. Thus it would be difficult to infer general principles from a patient-specific model.

There are few mathematical models of flow through the PDA in the literature. Many are electric circuit analogues of the full circulation, which have been adapted to include effects such as compliance, inertance, elastance and conservation equations (for example [13-15]). These cannot give insight into the local dynamics as there are no lengthscales involved. Other models couple electric analogues with onedimensional mass and momentum conservation equations $[16,17]$. The main drawback of both these and the previous models is that a large set of parameters is fitted from data. A third set of studies allows for the analysis of time-dependent pressure profiles by using impedance to monitor the propagation of the input signal away from the heart $[18,19]$. The PDA is treated as part of the aorta, so it is not possible to extract its specific influence or to predict the changes in the pulmonary system. In this paper we develop new two- and three-dimensional models of a region local to the shunt by assuming that the flow in this vicinity can be approximated as irrotational. We couple these models with an electric analogue of the global circulation dynamics, which effectively provides boundary conditions for the local geometry.

The driving pressure gradients in the aorta and pulmonary artery are dominated by their time-dependent components, whereas the velocity profiles have mean components of similar size to the fluctuating components. The dynamics in the two vessels are governed by two parameters, the Reynolds and Womersley numbers:

$$
\mathrm{Re}=\frac{\rho U R}{\mu} \approx 3030 \text { and } \alpha=R \sqrt{\frac{\rho \omega}{\mu}} \approx 16.5,
$$

where we use typical values as follows: blood density $\rho=1060 \mathrm{~kg} \mathrm{~m}^{-3}$ [20], dynamic viscosity of blood $\mu=0.0035 \mathrm{~kg} \mathrm{~m}^{-1} \mathrm{~s}^{-1}$ [21], cardiac period $T=0.7 \mathrm{~s}$ [22], corresponding to an angular frequency $\omega \approx 8.98 \mathrm{~s}^{-1}$, radius of the aorta $R=0.01 \mathrm{~m}$, and peak velocity in the aorta $U=1 \mathrm{~m} \mathrm{~s}^{-1}$ [23]. The Reynolds number of the flow is large, so inertial effects are dominant with viscous forces becoming important only near the walls. The Womersley number associated with the oscillating terms is also large, so if the mean component is neglected then the unsteady inertial forces dominate and the flow is essentially one of plug-like motion. 
The blood ejected from the heart into the aorta and pulmonary arteries during systole has little vorticity, so we can approximate it as irrotational. Furthermore, during diastole the valves are closed so that the velocity is much reduced, and thus there is negligible vorticity passed from one heartbeat to the next. For a pipe flow with an irrotational entry profile the flow becomes rotational due to viscous effects in boundary layers at the wall. These layers grow with increasing distance along the pipe over a lengthscale that is small compared to the lengthscale of downstream variation.

We argue that the PDA is sufficiently near the heart that the viscous boundary layers in the aorta and pulmonary artery are thin compared to the radii of these vessels and remain attached and well-behaved during the entire cardiac cycle. This enables us to obtain an approximation to the flow by modelling it as irrotational in the core of the vessel matched to viscous boundary layers at the walls. The assumption is easily justified for straight pipes, but the situation is less clear for arteries which curve in two or three dimensions. Riley [24] studied flow in a curved pipe that is started by the sudden imposition of a pressure gradient. He estimated that separation of the boundary layer occurs after a distance of approximately $3.98(2 \delta)^{-1 / 2}$ radii from the inlet, where $\delta$ is the ratio of the pipe radius to the radius of curvature of the pipe centreline. For the aorta where $\delta \approx 0.4$ [25] and $R \approx 0.01 \mathrm{~m}$, this formula suggests the flow is irrotational in the first $102^{\circ}$ of the bend. Moreover, the studies by Blyth and Mestel [26] and Zabielski and Mestel [27] suggest that the shedding of vortex pairs into the core of the fluid is delayed in helical pipes relative to toroidal pipes. Since the centreline of the aorta is non-planar, this could imply the flow remains irrotational further along the vessel. The position of the PDA varies between subjects, but it is usually situated at the saddle point of the aortic arch, corresponding to approximately $90^{\circ}$ into the vessel, justifying our assumption of irrotationality.

We scale velocities on the maximum velocity, $U$, distances on the aortic radius, $R$, times on the cardiac period, $T$, and pressures on $\rho U R T^{-1}$, so that the nondimensional form of the Navier-Stokes equations becomes

$$
\frac{\partial \mathbf{u}}{\partial t}+\frac{\operatorname{Re}}{\alpha^{2}}(\nabla \times \mathbf{u}) \times \mathbf{u}=-\nabla\left(p+\frac{\operatorname{Re}}{\alpha^{2}} \frac{|\mathbf{u}|^{2}}{2}\right)+\frac{1}{\alpha^{2}} \nabla^{2} \mathbf{u}
$$

where $\mathbf{u}$ and $p$ are the non-dimensional velocity and pressure flow profiles, and the non-dimensional numbers Re and $\alpha$ are given in Equation (1). If $\frac{1}{\alpha^{2}}$ and $\frac{\mathrm{Re}}{\alpha^{2}}$ are both small, then away from the walls $\frac{d \mathbf{u}}{d t} \simeq-\nabla p$. Even if $\frac{R e}{\alpha^{2}}$ cannot be neglected, for irrotational flows $\nabla \times \mathbf{u}=0$ and a similar result holds with the pressure modified by subtracting the term $\frac{\mathrm{Re}}{\alpha^{2}} \frac{|\mathbf{u}|^{2}}{2}$ from the potential flow approximation.

In this paper we investigate the haemodynamics by considering three idealised models of flow through a PDA. The choice of geometries is motivated by the available techniques for solving Laplace's equation analytically as well as the application of the solutions to study flows through shunts. For simplicity we neglect compliance and approximate the aorta and pulmonary artery as straight infinitely-long vessels. In Section 2.1 we study a two-dimensional geometry consisting of two parallel channels with a slit connecting them using complex potential methods. In Sections 2.2 and 2.3 we consider three-dimensional models: one consisting of two parallel touching cylinders with a point hole connecting them and the other consisting of a single 


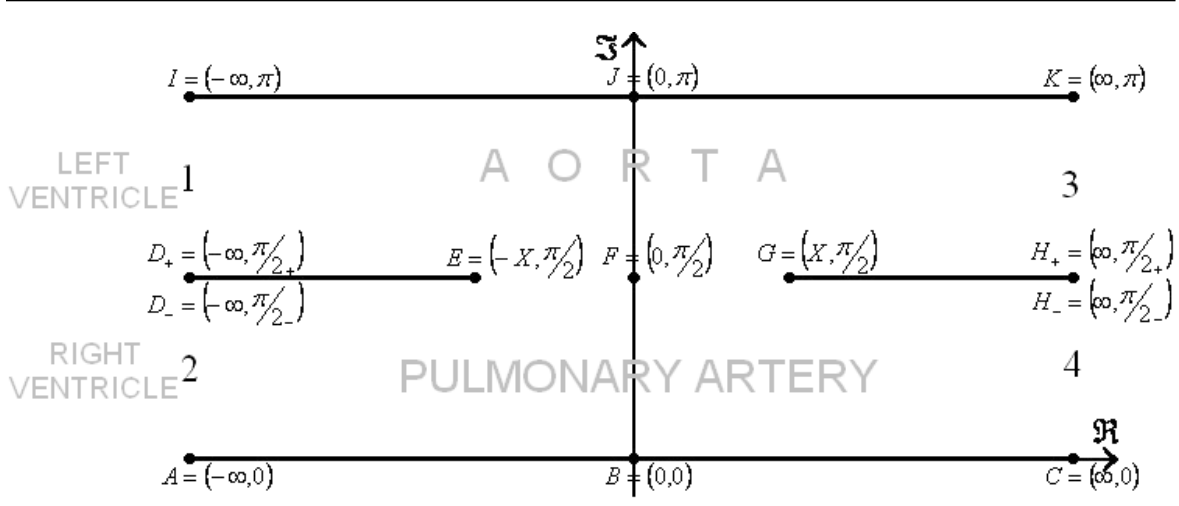

Fig. 2 Two-dimensional model considered in Section 2.1 in the complex z-plane, consisting of two infinite channels of width $\pi / 2$ representing the aorta (top) and pulmonary artery (bottom) with the heart connected to inlets 1 and 2, and the systemic and pulmonary circulations to outlets 3 and 4 . The shunt has length $2 X$.

cylinder divided in two by flat plates with a gap between them. We construct solutions for both of these models using series expansions of analytical functions.

\section{Mathematical model of the PDA and solution for the spatial dependence}

The high-frequency time dependence in the aorta and pulmonary artery, as well as the irrotational flow profiles at the ventricles, enable us to approximate the haemodynamics near a PDA using potential flow analysis. Assuming that the vorticity is confined to thin boundary layers during the cardiac cycle and that there is no vorticity diffusion or separation into the core flow, the velocity and pressure of the flow satisfy

$$
\nabla \cdot \mathbf{u}=0 \quad \text { and } \quad \frac{\partial \mathbf{u}}{\partial t}=-\nabla P
$$

where $P=p+\frac{\operatorname{Re}}{\alpha^{2}} \frac{|\mathbf{u}|^{2}}{2}$ is the static plus dynamic pressure. So, the potential $\phi$ satisfies

$$
\mathbf{u}=\nabla \phi, \quad \nabla^{\mathbf{2}} \phi=\mathbf{0} \quad \text { and } \quad \frac{\partial \phi}{\partial t}+P=f(t)
$$

where $f$ is an arbitrary function. Equations (4) are to be solved together with no penetration boundary conditions $\mathbf{n} \cdot \nabla \phi=0$ at the walls and suitable inflow and outflow conditions. We seek a separable solution of the form $\mathbf{u}(\mathbf{x}, t)=\sum_{n} \mathbf{u}_{n}(\mathbf{x}) T_{n}(t)$ or, correspondingly, $\phi(\mathbf{x}, t)=\sum_{n} \phi_{n}(\mathbf{x}) T_{n}(t)$. The functions $T_{n}$ are set by the boundary conditions at the vessels' inlets and outlets. In Sections 2.1-2.3 we calculate the set of $\phi_{n}$ for each geometry under consideration.

\subsection{Two channels with a shunt between them}

The geometry is shown in Figure 2 and consists of two parallel doubly infinite channels connected by a slit of length $2 X$. The ends of the vessels are labelled 1 to 4 as 


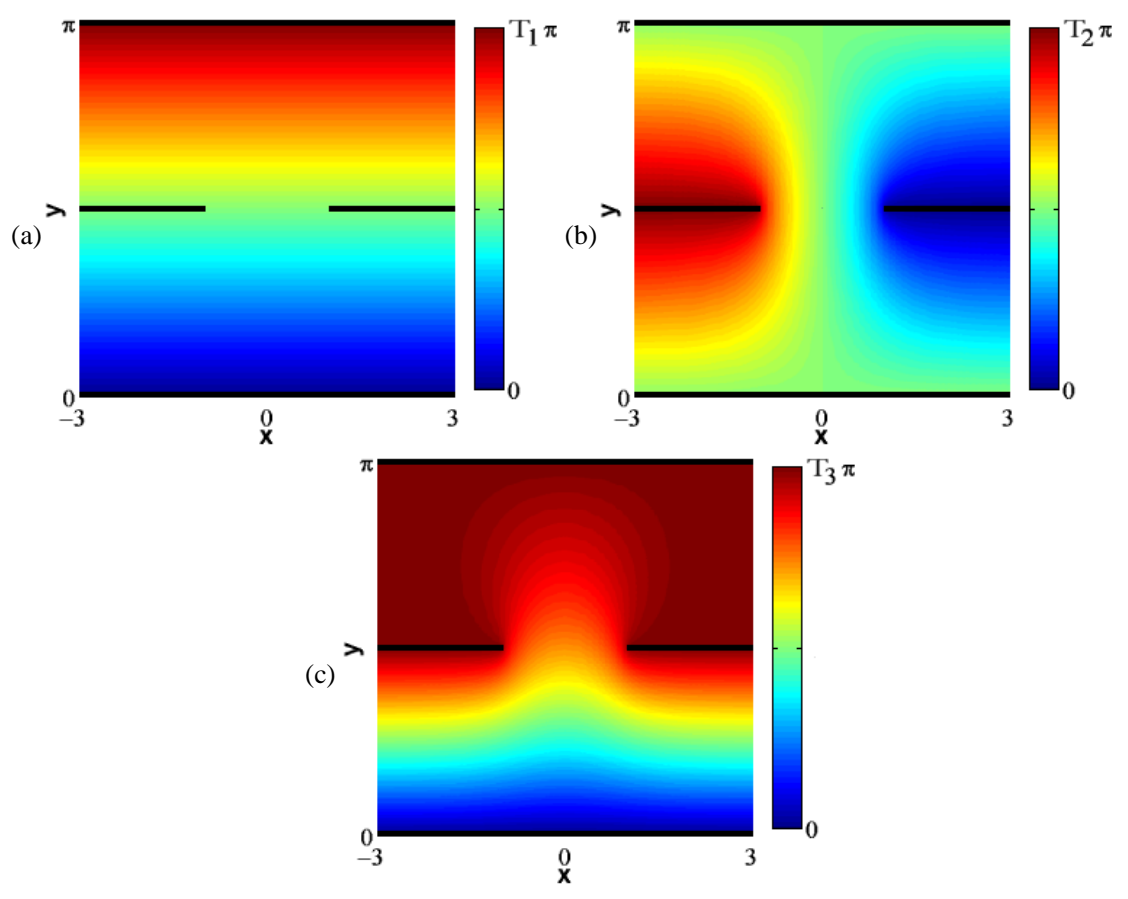

Fig. 3 Contour plots of the non-dimensional stream functions (a) $\psi_{1}(z)=\mathfrak{I}\left(w_{1}(z)\right)$, (b) $\psi_{2}(z)=\mathfrak{I}\left(w_{2}(z)\right)$ and (c) $\psi_{3}(z)=\mathfrak{I}\left(w_{3}(z)\right)$ in the complex $z$-plane. The non-dimensional shunt width is $2 X=2$ in all plots.

shown; we use corresponding superscripts to denote the values of quantities evaluated there. It can be shown that at each inlet or outlet, the only solution of (4a) that satisfies the boundary conditions and does not blow up exponentially fast is uniform flow parallel to the walls, and thus we only need to specify the velocity magnitudes $U_{1,2,3,4}$ at the inlets and outlets, with $U_{1}+U_{2}=U_{3}+U_{4}$ due to conservation of mass.

In two dimensions Equations (3) imply that there exist both a velocity potential $\phi_{n}(x, y)$ and a stream function $\psi_{n}(x, y)$ associated with each velocity $\mathbf{u}_{\mathbf{n}}$ such that

$$
\mathbf{u}_{\mathbf{n}}(x, y)=\nabla \phi_{n}(x, y)=\nabla \times\left(\psi_{n}(x, y) \mathbf{k}\right)
$$

where $\mathbf{k}$ is the unit vector perpendicular to the two-dimensional plane. We solve for the complex potentials, $w_{n}=\phi_{n}+i \psi_{n}$. The real and imaginary parts of $w_{n}$ satisfy the Cauchy-Riemann equations and thus each $w_{n}$ is analytic. The four inlet and outlet boundary conditions together with the restriction imposed by conservation of mass imply there are at most three linearly independent solutions for $w_{n}$, which are denoted $w_{1}, w_{2}$ and $w_{3}$. We present analytical expressions for a particular set (Figure 3) 

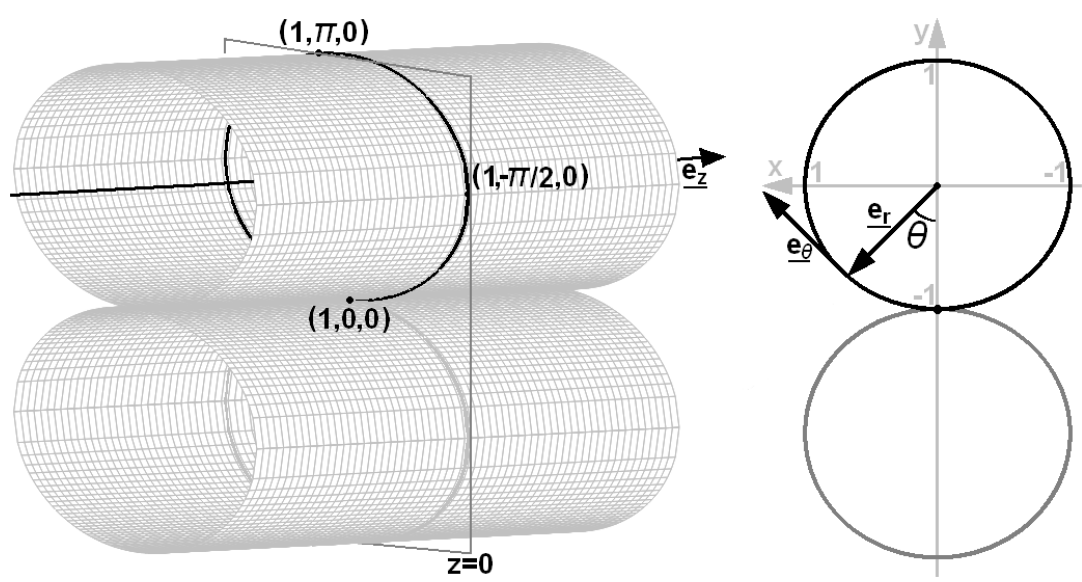

Fig. 4 Left: Geometry of the system considered in Section 2.2 consisting of two infinite cylinders (representing the aorta on top and the pulmonary artery on the bottom) connected by a point singularity (representing the PDA). The heart is connected to the two inlets $z \rightarrow-\infty$ and the systemic and pulmonary circulations to the outlets $z \rightarrow \infty$. Right: Cross-sectional view and a diagram of the cylindrical coordinates.

$$
\begin{aligned}
& w_{1}=T_{1} z, \\
& w_{2}=T_{2} \ln \left(-i \frac{\sinh X+\cosh z-\sqrt{\sinh ^{2} X+\cosh ^{2} z}}{\sinh X-\cosh z-\sqrt{\sinh ^{2} X+\cosh ^{2} z}}\right), \\
& w_{3}=T_{3} \ln \left(\frac{e^{2 z}-1-\sqrt{2 e^{2 z}(\cosh (2 X)+\cosh (2 z))}}{2 \cosh (X)}\right),
\end{aligned}
$$

where $z=x+i y$. We obtained these by finding conformal mappings, which send the boundaries of the geometry in Figure 2 to the unit circle, that were then used to construct each complex potential profile using the Milne-Thomson Circle Theorem. This was done using a convolution of hyperbolic maps and Schwarz-Christoffel transformations. Since the governing equations are linear we can superpose these solutions to find the general solution $w(\mathbf{x}, t)=\sum_{n=1}^{3} w_{n}(\mathbf{x}) T_{n}(t)$, which can be used to calculate the velocity and pressure in the core flow. We also estimate the wall shear stress by introducing Stokes boundary layers (of width $\approx 1 / \alpha$ times the width of each channel) at all rigid walls. It is proportional to the slip velocity of the irrotational flow.

The velocity profile behaves like $r^{-1 / 2}$ near each edge of the shunt, where $r$ is the distance away from the corresponding edge. This singularity behaviour depends on the angle of the corner that the flow needs to turn. For example, if the shunt is modelled as a rectangle (i.e. if the angle of the bend is 270 degrees rather than 360 degrees) then the velocity singularities would behave as $r^{-1 / 3}$ [28]. When viscosity is reintroduced, the Stokes layers of thickness $\alpha^{-1}$ ensure that the shear stress at the corners is finite and of the order $\alpha^{1 / 2}$ and $\alpha^{1 / 3}$ for the two corners respectively. 


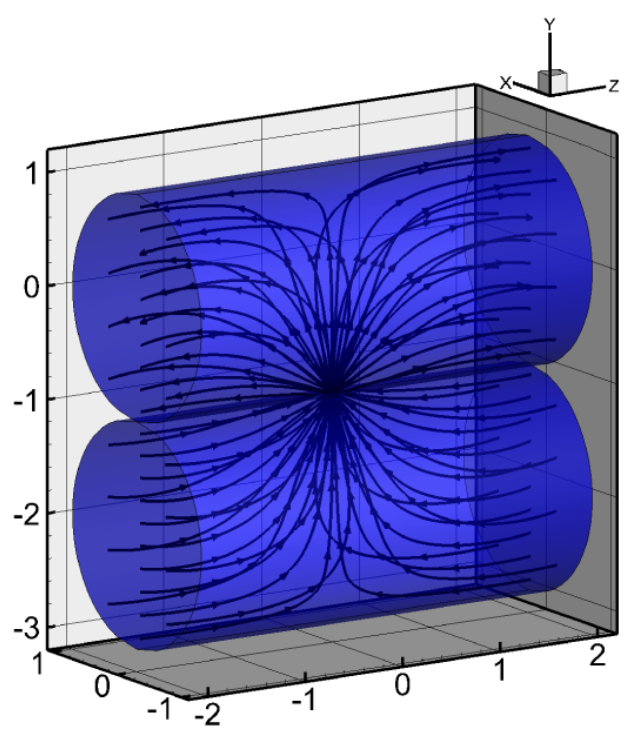

Fig. 5 Streamlines of the flow corresponding to the velocity potential solution in Equation (10) for any positive value of $Q_{3}$. If $Q_{3}$ is negative the arrows would be pointing in the opposite directions. The coordinates $x$ and $y$ are defined by $x=r \sin \theta$ and $y=-r \cos \theta$ respectively.

\subsection{Two cylinders with a point singularity between them}

We consider two doubly infinite cylinders of radius 1 linked by a point singularity, see Figure 4 . We work in cylindrical coordinates $(r, \theta, z)$ natural to the top cylinder with the singularity at the point $(1,0,0)$. As in Section 2.1 the flow must be uniform and purely axial at each of the four cylinder ends, so the boundary conditions are specified by the inlet and outlet velocities, $U_{1,2,3,4}$, and mass conservation implies there are three linearly independent solutions. These can be written in terms of their velocity potentials, $\phi_{k}(\mathbf{x}), k=1 . .3$, each of which satisfies Laplace's equation, $\nabla^{2} \phi_{k}=0$, as before. However, since the flow is fully three-dimensional there are no corresponding stream functions. As before we apply no penetration boundary conditions at the walls.

There are two linearly independent trivial solutions, which are spanned by uniform flow in one pipe and no flow in the other, given by

$$
\phi_{1}(\mathbf{x})=\left\{\begin{array}{ll}
z & \text { in top cylinder } \\
0 & \text { in bottom cylinder }
\end{array} \text { and } \phi_{2}(\mathbf{x})= \begin{cases}0 & \text { in top cylinder } \\
z & \text { in bottom cylinder }\end{cases}\right.
$$

The third solution drives fluid through the point singularity. We choose a flow that is symmetric in both the plane $z=0$ and in the common tangent plane of the two cylinders. Due to symmetry, we calculate the solution in the top cylinder and find the solution in the other by reflection. In these coordinates the boundary condition is $\frac{\partial \phi_{3}}{\partial r}=Q_{3} \delta(z) \delta(\theta)$ at $r=1$. The constant $Q_{3}$ represents the flux through the point singularity and thus we also stipulate that the uniform velocity at each inlet or outlet is $\frac{\partial \phi_{3}}{\partial z}= \pm \frac{Q_{3}}{2 \pi}$ depending on the direction of the flow. We use a Fourier transform and 
(a)

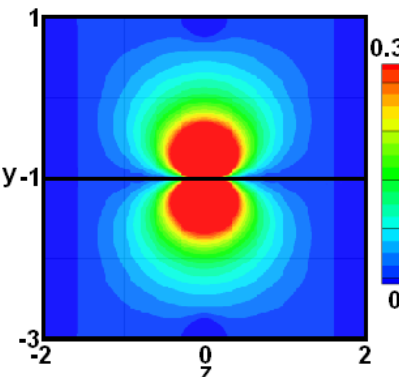

(c)

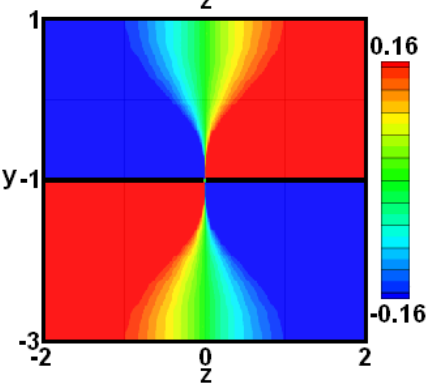

(b)

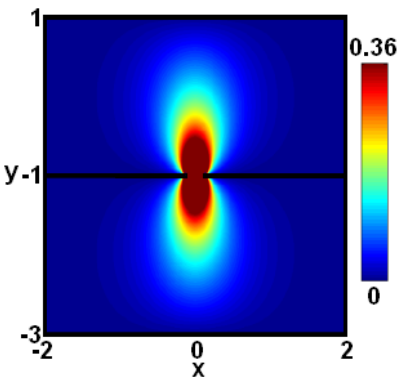

(d)

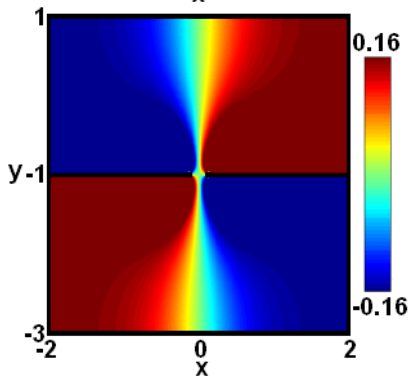

Fig. 6 Comparison between the non-zero velocity components in the $x=0$ plane of the three dimensional model (Section 2.2) and the equivalent two-dimensional profiles (Section 2.1) if the shunt width equals 0.2 . Contours of (a) $v$ and (c) $w$ associated with the velocity potential in Equation (10) and of (b) $v$ and (d) $u$ associated with the complex potential in Equation (7) where $T_{2}=1 / 2 \pi$.

Fourier series to find the particular solution to Laplace's equation

$$
\phi_{3}(r, \theta, z)=\frac{Q_{3}}{2 \pi} \int_{-\infty}^{\infty}\left[\frac{I_{0}(k r)}{2 \pi k I_{0}^{\prime}(k)}-\frac{1}{\pi k^{2}}+\sum_{n=1}^{\infty} \frac{\cos (n \theta) I_{n}(k r)}{\pi k I_{n}^{\prime}(k)}\right] \exp (i k z) d k
$$

where $I_{n}$ is the modified Bessel function of the first kind of order $n$. The term $-1 / \pi k^{2}$ is included to remove the singularity at $k=0$ in the square brackets. The approximate streamlines based on using the first 12 terms of the truncation and performing the integral over the range $[-100,100]$ are shown in Figure 5. The Cartesian coordinates are defined by $x=r \sin \theta$ and $y=-r \cos \theta$, and we write the velocity as $(u, v, w)$. The plane $x=0$ containing the centrelines of both cylinders is a plane of symmetry, and no streamlines cross this plane. The velocity profiles within this plane when $Q_{3}=1$ and the equivalent profiles from the two-dimensional model considered in Section 2.1 for a shunt size one tenth of the width of the channel are compared in Figure 6. Note that the $z$-coordinate and therefore the $w$-velocity component in this section correspond to the $x$-coordinate and thus the $u$-velocity component in Section 2.1. The velocity component $u$ in the three-dimensional model is exactly zero by construction in the chosen plane $x=0$. There is good agreement of the other two velocity components between the two models with slowest convergence of the truncation errors in the three-dimensional model along the curve $\{r=1, z=0\}$. We note singularities at the shunt edges in all four computed profiles; however, the scales in the plots have been restricted so that the dynamics away from the singularities could be compared. 

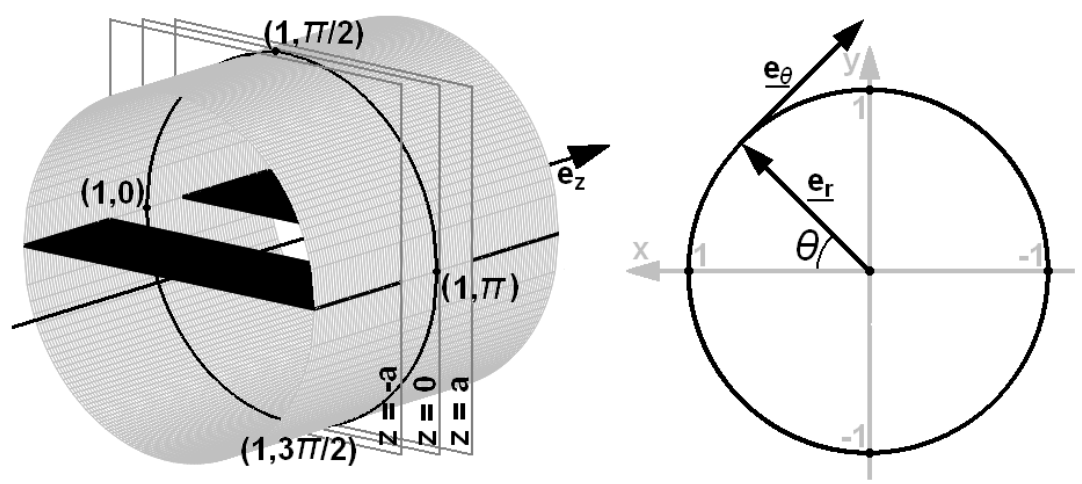

Fig. 7 Left: Geometry of the system considered in Section 2.3 consisting of two infinite semi-cylinders (representing the aorta on top and the pulmonary artery on the bottom) connected by a rectangular shunt of non-dimensional size $1 \times 2 a$ (representing the PDA). The heart is connected to the two inlets $z \rightarrow-\infty$ and the systemic and pulmonary circulations to the outlets $z \rightarrow \infty$. Right: Cross-sectional view and a diagram of the cylindrical coordinates.

The full solution for the velocity potential is $\phi(\mathbf{x}, t)=\sum_{n=1}^{3} \phi_{n}(\mathbf{x}) T_{n}(t)$, which can be used to estimate the three-dimensional dynamics in the aorta and pulmonary artery. However, this model is limited in capturing the dynamics near the shunt; in particular, a Stokes boundary layer approximation at the rigid wall is inconsistent with the imposed point singularity there. This motivated us to consider another threedimensional geometry where the shunt is modelled as a two-dimensional surface.

\subsection{Two semi-cylinders joined by a plate}

The third, and final, idealised geometry we consider consists of a cylinder that is divided by a plate with a rectangular gap (Figure 7). We use cylindrical coordinates natural to this cylinder with $r=1$ corresponding to the surface of the cylinder and the two rigid walls between the semi-cylinders described by $\{\theta=0, \pi, z \geq a\}$ and $\{\theta=$ $0, \pi, z \leq-a\}$. We use Cartesian coordinates defined by $x=r \cos \theta$ and $y=r \sin \theta$. The shunt is a rectangle of width 1 in the $x$-direction and width $2 a$ in the $z$-direction.

We seek a set of three linearly independent flow profiles as before. Each is described by a velocity potential $\phi_{n}$ that satisfies Laplace's equation, no-flux boundary conditions on the cylinder described by $\frac{\partial \phi_{n}}{\partial r}=0$ at $r=1$, no-flux boundary conditions on the plate between the two vessels and $\frac{1}{r} \frac{\partial \phi_{n}}{\partial \theta}=0$ at $\theta=0$ or $\pi$ when $|z| \geq a$, and uniform flow at the inlets and outlets of the geometry. We construct solutions for a particular set of three such solutions by partitioning the geometry into subregions and considering expansions motivated by the general solution of Laplace's equation in cylindrical coordinates in each subregion. The choice of set is motivated by the number of planes of symmetries we can impose on each solution to simplify 
the derivations. The expansions and partitions we consider are

$$
\begin{aligned}
& \phi_{1}(\mathbf{x})=T_{1} z \\
& \phi_{2}(\mathbf{x})=\left\{\begin{array}{ll}
\sum_{n=0}^{\infty} \sum_{k=1}^{\infty} a_{k}^{n} J_{2 n+1}\left(\mu_{k}^{2 n+1} r\right) \sin ((2 n+1) \theta) \cosh \left(\mu_{k}^{2 n+1} z\right) & r \in[0,1], \theta \in[0, \pi], z \in[0, a] \\
T_{2} z+\sum_{n=0}^{\infty} \sum_{k=1}^{\infty} b_{k}^{n} J_{2 n}\left(\mu_{k}^{2 n} r\right) \cos (2 n \theta) e^{-\mu_{k}^{2 n}(z-a)} & r \in[0,1], \theta \in[0, \pi], z \in[a, \infty)
\end{array}\right) \\
& \phi_{3}(\mathbf{x})=\left\{\begin{array}{cc}
B_{0}^{0} z+\sum_{n=0}^{\infty} \sum_{k=1}^{\infty} A_{k}^{n} J_{2 n+1}\left(\mu_{k}^{2 n+1} r\right) \sin ((2 n+1) \theta) \sinh \left(\mu_{k}^{2 n+1} z\right) \\
+\sum_{n=0}^{\infty} \sum_{k=1}^{\infty} B_{k}^{n} J_{2 n}\left(\mu_{k}^{2 n} r\right) \cos (2 n \theta) \sinh \left(\mu_{k}^{2 n} z\right) & r \in[0,1], \theta \in[0,2 \pi], z \in[0, a] \\
T_{3} z+\sum_{n=0}^{\infty} \sum_{k=1}^{\infty} C_{k}^{n} J_{2 n}\left(\mu_{k}^{2 n} r\right) \cos (2 n \theta) e^{-\mu_{k}^{2 n}(z-a)} & r \in[0,1], \theta \in[\pi, 2 \pi], z \in[a, \infty) \\
\sum_{n=0}^{\infty} \sum_{k=1}^{\infty} D_{k}^{n} J_{2 n}\left(\mu_{k}^{2 n} r\right) \cos (2 n \theta) e^{-\mu_{k}^{2 n}(z-a)} & r \in[0,1], \theta \in[0, \pi], z \in[a, \infty)
\end{array}\right.
\end{aligned}
$$

where the constant

$$
\mu_{k}^{n} \text { is the } k^{\text {th }} \text { positive root in ascending order of } J_{n}{ }^{\prime}(\mu)=0 .
$$

The unknown constants in these expansions can be found by considering the set of equations that ensure continuity of each velocity potential solution and its first derivatives across any subregion boundary or partition. We then reduce the number of equations using four semi-orthogonal identities of the circular and Bessel functions:

$$
\int_{0}^{\pi} \int_{0}^{1} r J_{n}\left(\mu_{k}^{n} r\right) \sin (n \theta) J_{N}\left(\mu_{K}^{N} r\right) \sin (N \theta) d r d \theta=\frac{\pi \delta_{n N} \delta_{k K}}{2} \int_{0}^{1} r\left(J_{n}\left(\mu_{k}^{n} r\right)\right)^{2} d r
$$

if both $n$ and $N$ are odd (15)

and three similar expressions obtained by symmetry. We use the notation $\delta_{i j}$ for the Kronecker delta function. For example, the expansion of the solution $\phi_{3}$ in Equation (13) requires four equations of continuity evaluated at $z=a$ : two in the top semicylinder that need to be satisfied $\forall r \in[0,1], \theta \in[0, \pi]$ and two in the bottom semicylinder that need to be satisfied $\forall r \in[0,1], \theta \in[\pi, 2 \pi]$. Using the semi-orthogonal relationships we can represent each unknown $B_{k}^{n}, C_{k}^{n}$ and $D_{k}^{n}$ as an infinite linear combination of the unknowns $A_{k}^{n}$, and thus the number of equations we need to solve reduces by a factor of four. The problem is simplified to a linear system of infinite size for the unknowns $A_{k}^{n}, k=1 . . \infty, n=0 . . \infty$ and progress can be made by truncating the infinite series and observing that the terms multiplying each constant $A_{k}^{n}$ in Equation (13) decrease as $k$ and $n$ increase. The same technique was applied to obtain the two sets of constants in the expansions in Equation (12).

Figure 8 shows the streamlines that correspond to the solutions $\phi_{2}$ and $\phi_{3}$ for the truncations $k=1 . .8$ and $n=1 . .14$ and the specific shunt width $2 a=2$. These were obtained by differentiating the analytical expression for each velocity potential, calculating the velocity field for a mesh consisting of $40 \times 40 \times 80$ points and integrating numerically between points. The plots, and in particular the smooth streamlines across the interfaces at $z= \pm 1$, suggest that the matching between 


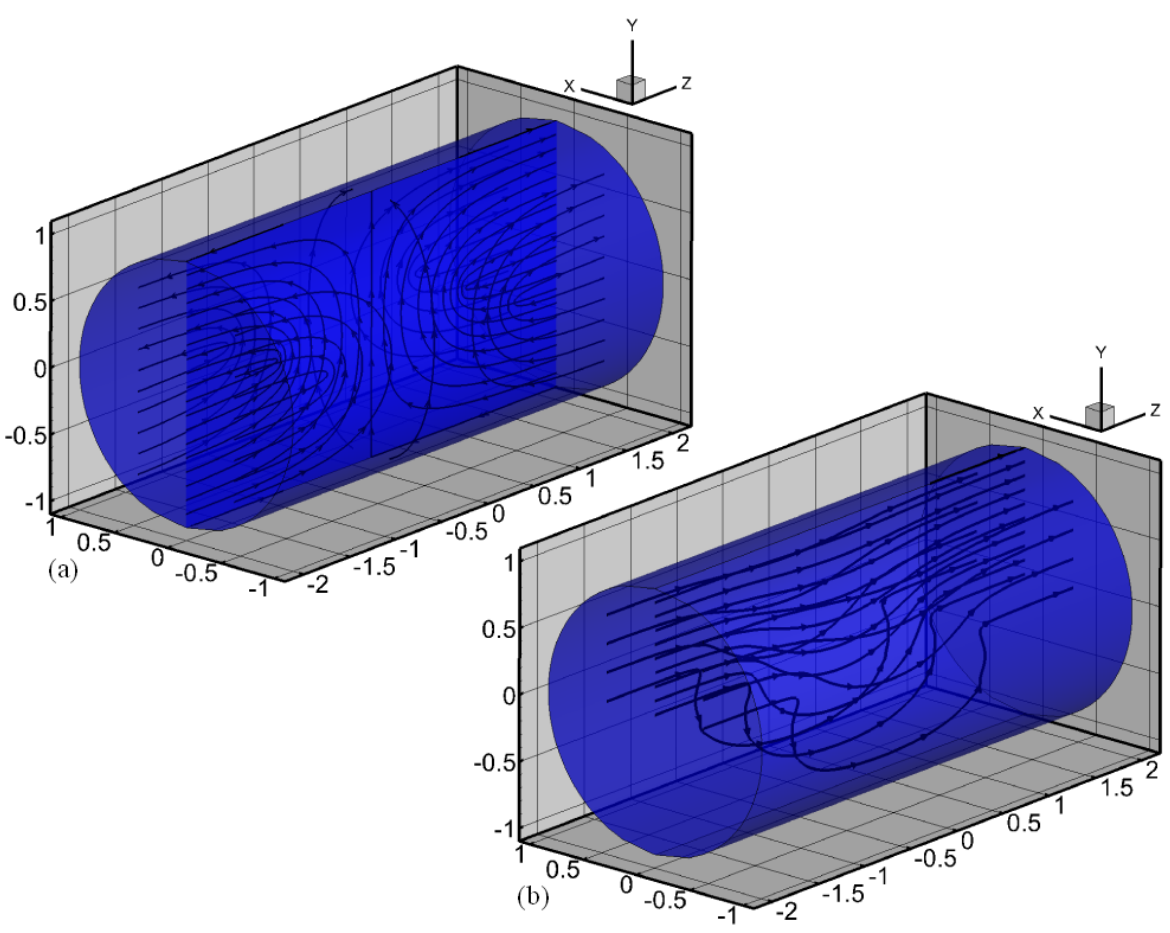

Fig. 8 Streamlines of the flows corresponding to the velocity potential solution $\phi_{2}$ (a) and $\phi_{3}$ (b) from Equations (12) and (13) respectively. The constants $T_{2}, T_{3}$ and $a$ are all chosen to equal one. The coordinates $x$ and $y$ are defined by $x=r \cos \theta$ and $y=r \sin \theta$. The two rigid walls in the plane $y=0$ and bounded by $z \in[1, \infty)$ and $z \in(-\infty,-1]$ can be identified by the streamlines that curve round them in both plots.

partitions is sufficiently smooth despite the truncations of the series in the analytical solutions. The main behaviour that cannot be fully captured by this method is the velocity singularity along the sharp edges of the shunt. However, the two-dimensional model that we developed in Section 2.1 tells us that this singularity behaves like $r^{-1 / 2}$ near the edges where $r$ is the shortest distance to the edges. The flow described by $\phi_{1}$ is uniform in the $z$-direction and is thus trivial. The velocity fields in the midplane $x=0$ of all three solutions compare very well with the two-dimensional equivalents in Section 2.1.

A general solution can be constructed as before using a linear combination of the three flows $\phi(\mathbf{x}, t)=\sum_{n=1}^{3} \phi_{n}(\mathbf{x}) T_{n}(t)$. The functions $T_{n}(t)$ determine the flow profile at every time step. We next derive a model for the global flow dynamics in the pulmonary and systemic circulations in order to estimate possible time-dependent functions for all three spatial models that we have considered so far. 


\section{Time dependance and results}

The models of Sections 2.1, 2.2 and 2.3 each depend on the instantaneous values of the fluxes $U_{1}, U_{2}, U_{3}$ and $U_{4}$. In practice these values are time-dependent and are determined by other factors in the circulation. We model these using bulk parameters derived from an electric circuit analogue. This is used to provide boundary conditions for the flux at the inlets and outlets of each geometry. The circulation compartments are modelled as resistors or capacitors joined together by vessels in a closed circuit. Here we describe the coupling between the two-dimensional geometry in Section 2.1 and such an electric analogue but the method can be easily adapted for the other models as well: the only change is that the cross-sectional area of each vessel needs to be chosen such that the fluxes and velocity profiles at the inlets and outlets match with the available physiological data.

Let $U_{n}(t)$ and $P_{n}(t)$ be the non-dimensional horizontal velocity and pressure evaluated at the $\mathrm{n}^{\text {th }}$ inlet or outlet of the geometry. We seek to find eight equations to complete the system. Conservation of mass

$$
U_{1}(t)+U_{2}(t)=U_{3}(t)+U_{4}(t),
$$

modified Ohm's law relationships for the downstream dynamics

$$
\begin{aligned}
& P_{3}=\mu\left(P_{v}+R_{3} U_{3}\right) \\
& P_{4}=\mu\left(P_{p}+R_{4} U_{4}\right)
\end{aligned}
$$

and the Bernoulli equation

$$
\frac{\partial U_{1}}{\partial t}+P_{1}(t)=\frac{\partial U_{2}}{\partial t}+P_{2}(t)=\frac{\partial U_{3}}{\partial t}+P_{3}(t)=\frac{\partial U_{4}}{\partial t}+P_{4}(t)
$$

provide six equations for the eight unknowns. The constants $P_{v}$ and $P_{p}$ are time averages of the pressure in the systemic venous and systemic pulmonary arterial circulations, whereas $R_{3}$ and $R_{4}$ are the resistances in these circulations. The constant $\mu$ is the ratio between the aortic radius and the distance between the left ventricle and the shunt, and could be absorbed into the other parameters.

Two further conditions are required, relating the input pressures and fluxes at the ventricles. Here, we assume that the heart, blood vessels and lungs in some asymptomatic patients compensate in such a way that the cardiac output produced by the ventricles is the same as in healthy adults. This is equivalent to prescribing the spatially-uniform velocity at the ventricles. We chose typical profiles from different patients using measurements courtesy of Dr. Justin Davies at Saint Mary's Hospital, London. We normalised these data to be consistent with our model and prescribed zero flow at the ventricles during diastole. The velocities at the left and right ventricles $U_{1}(t)$ and $U_{2}(t)$ are shown in Figure 9. They complete the system of equations needed to solve for all horizontal velocities $U_{n}(t)$ and pressure profiles $P_{n}(t)$ at the inlets and outlets. In these simulations we use the following dimensional parameters: the length of the shunt is $L=0.009 \mathrm{~m}$ [30], the distance from the aortic root to the centre of the shunt is $d_{v d}=0.075 \mathrm{~m} \mathrm{[31]}$, the resistances in the systemic and in the the pulmonary circulations are $R_{3}=1050 \times 10^{5} \mathrm{~kg} \mathrm{~s}^{-1} \mathrm{~m}^{-4}\left(1050\right.$ dynes s cm$\left.{ }^{-5}\right)$ 

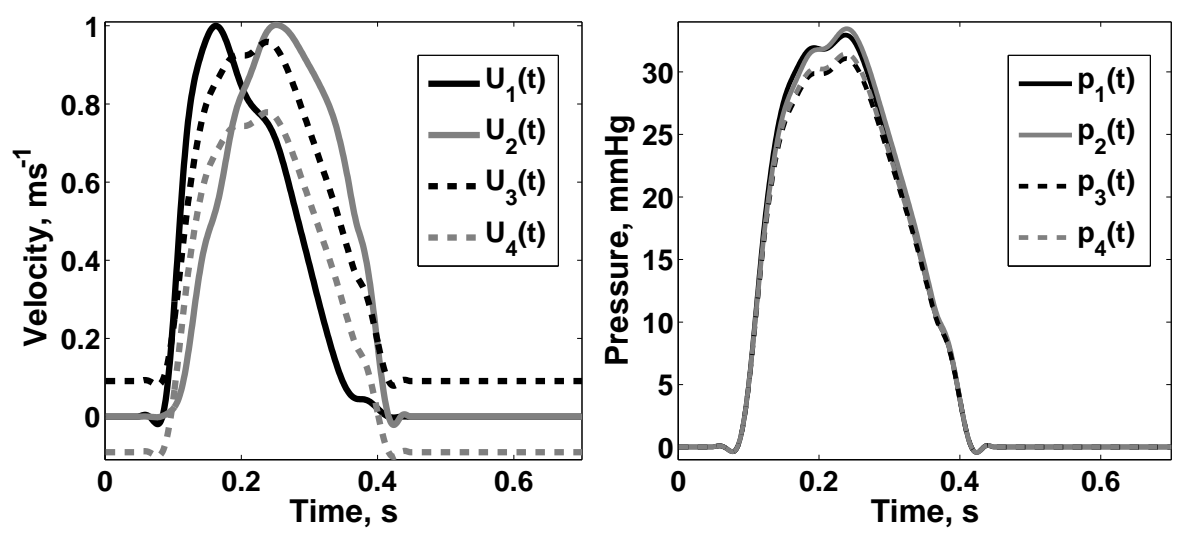

Fig. 9 Predicted velocities (left) and pressures (right) in the ascending aorta (black line) and in the pulmonary artery (grey line) upstream (solid line) or downstream (dashed line) from the shunt in an asymptomatic PDA patient whose heart produces the same cardiac output as a healthy person. The pressure integration constant is chosen to be such that the pressure at the ventricles is zero during diastole.

[32] and $R_{4}=150 \times 10^{5} \mathrm{~kg} \mathrm{~s}^{-1} \mathrm{~m}^{-4}\left(150\right.$ dynes $\left.\mathrm{s} \mathrm{cm}^{-5}\right)$ [32], the mean systemic venous pressure is $P_{v}=8 \mathrm{mmHg}$ [33] and the mean systemic pulmonary arterial pressure is $P_{p}=15 \mathrm{mmHg}$ [34]. These values were non-dimensionalised using the characteristic values given just before Equation (2) and substituted into the nondimensional Equations (17-18).

It should be remembered that the potential pressure $P_{n}(t)$ is related to the physiological pressure $p_{n}$ by the relationship $p_{n}(t)=P_{n}(t)-\frac{\mathrm{Re}}{\alpha^{2}} \frac{\left|U_{n}(t)\right|^{2}}{2}$ as discussed after Equation (2). An integration constant can be added to the profiles, which is chosen so that the pressure at the ventricles during diastole is zero. The velocity and pressure at the two inlets and two outlets are shown in Figure 9. The time-dependent constants $T_{n}(t)$ that define the flow at all points in the cardiac cycle can be found from the velocity profiles. The mathematical models in Section 2 can now be used to analyse the flow dynamics in the vicinity of the shunt.

\subsection{Streamlines and flux}

Plots of the dimensional stream function $\psi(x, y, t)$ at four different times during the heartbeat are shown in Figure 10. The contours in these plots coincide with the streamlines at those particular times and the difference in value between two points is the flux through a line connecting these points.

Figure 10(a) at time $t=0 \mathrm{~s}$ represents a typical point during diastole. Our model predicts that there is backflow from the pulmonary artery into the aorta during diastole due to the lower pressure in the systemic vascular circulation compared to the pulmonary circulation $\left(P_{v}<P_{p}\right)$. This flow is relatively small: its magnitude is one tenth of the maximum flow during systole. However, it is important in terms of blood distribution as de-oxygenated blood bypasses the lungs and is sent to the systemic 


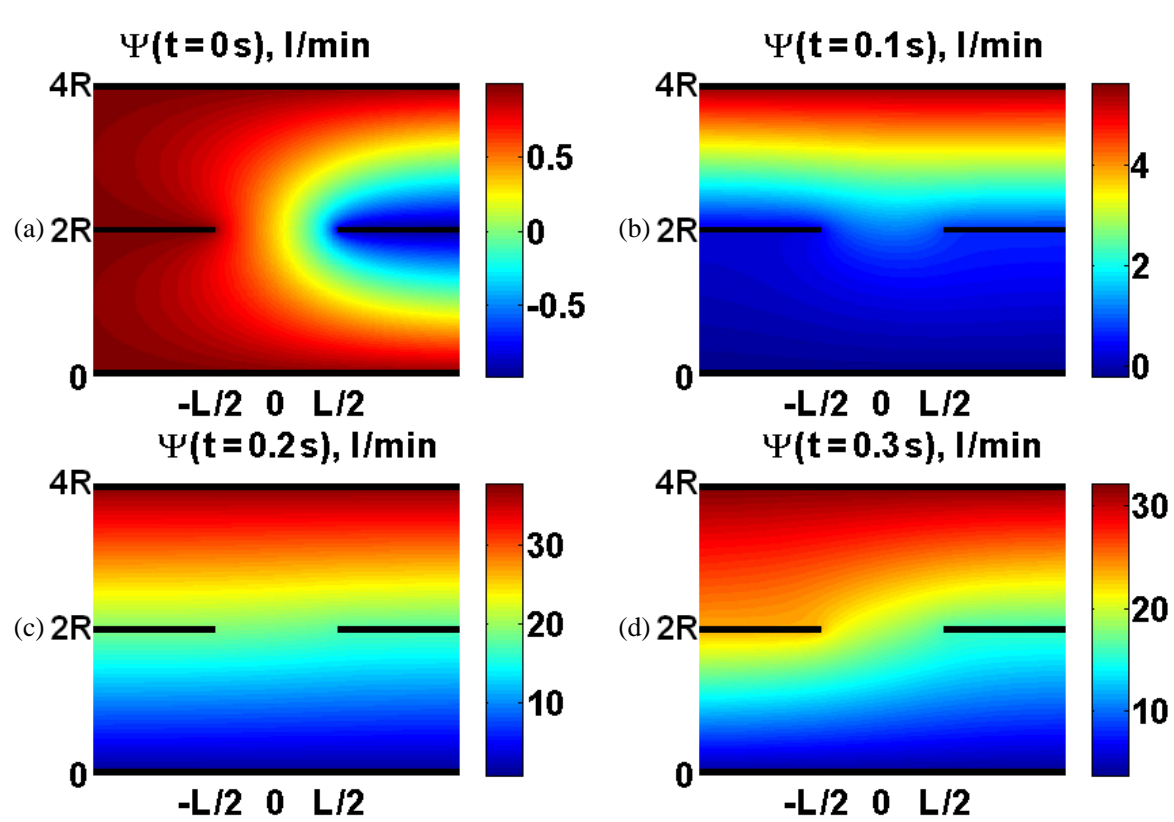

Fig. 10 Stream function $\psi$, measured in litres per minute, in a two-dimensional cross section of the aorta (top channel), the pulmonary artery (bottom channel) and a PDA at times (a) $t=0 \mathrm{~s}$, (b) $t=0.1 \mathrm{~s}$, (c) $t=0.2 \mathrm{~s}$ and (d) $t=0.3 \mathrm{~s}$. The two channels are of width $2 R=0.02 \mathrm{~m}$ and the shunt width is $L=0.009 \mathrm{~m}$.

system, which could potentially have a huge significance on the well-being of a PDA patient. Such a backflow has been detected in PDA patients $[35,36]$.

In addition, our model predicts that during systole the flow volume leaving the aorta is larger than that leaving the pulmonary artery, as can be seen from the area under the curves $U_{3}(t)$ and $U_{4}(t)$ in Figure 9. This suggests that either the lungs receive a smaller volume to oxygenate in asymptomatic PDA patients than in healthy adults, or that the heart compensates for such a loss in oxygen by ejecting higher cardiac volumes. Figures 10 (c) and $10(\mathrm{~d})$ show the stream function at two typical time points during systole. At time $t=0.2 \mathrm{~s}$, there is almost no flow being shunted through the PDA. However, at time $t=0.3 \mathrm{~s}$, a flux of approximately $7 \operatorname{lmin}^{-1}$ bypasses the lungs and is sent to the systemic circulation. If this happens during every cardiac cycle then the cardiac output is likely to increase to compensate for the loss in oxygen perfusion. It is observed in the literature that PDA patients can exhibit similar physical exertion at rest as do healthy adults during exercise. This, in turn, can explain why PDA patients can appear asymptomatic and yet develop serious conditions with time: it is not natural for the body to work at high rates continuously with no recovery period.

The specific time point $t=0.1 \mathrm{~s}$ in Figure 10(b) happens to be during the short transition between diastole and systole. In particular, it is at a time when the left ventricle is pumping a larger output (approximately $4.5 \mathrm{lmin}^{-1}$ ) compared to the right one (approximately $0.5 \mathrm{lmin}^{-1}$ ) due to the small time lag between the contraction of the left and right ventricles. A small but considerable amount of blood (approximately 


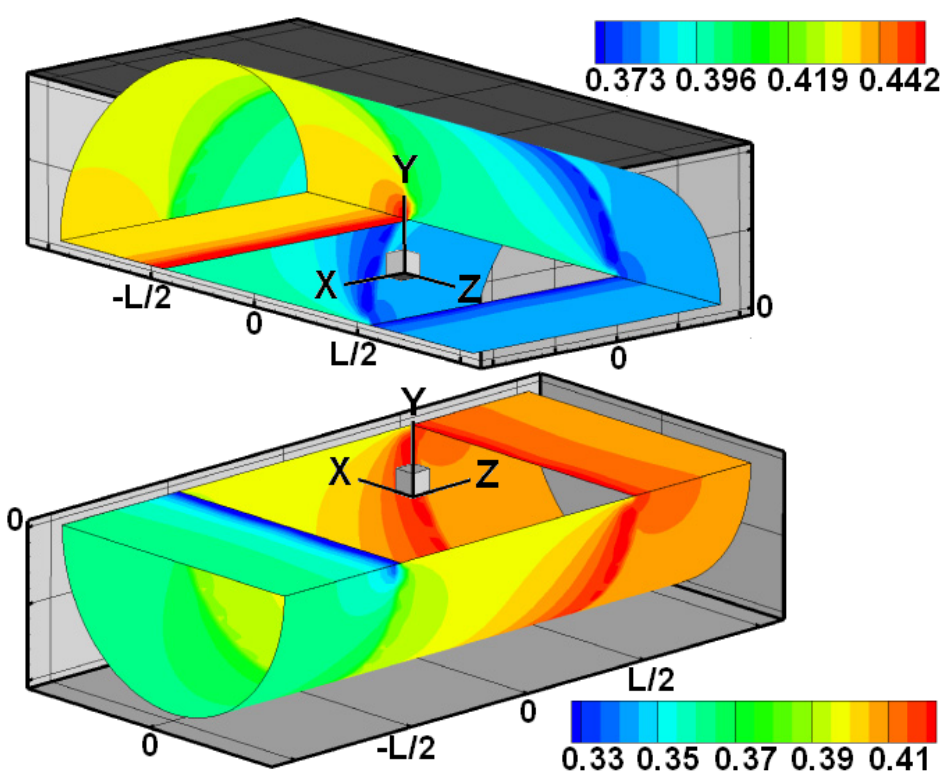

Fig. 11 Shear stress $\tau$ at time $t=0.16 \mathrm{~s}$, measured in pascals, in a three-dimensional geometry of two semi-cylinders representing the aorta (left) and the pulmonary artery (right) joined by a rectangular PDA joining them. The two semi-cylinders are of height $\sqrt{2} R \approx 0.014 \mathrm{~m}$, the shunt is of size $0.009 \mathrm{~m}$ by $\approx 0.028$ $\mathrm{m}$. The $z$-coordinate is stretched by a factor of $\approx 3.14$ with respect to the $x$ and $y$-coordinates in the figures.

$0.2 \operatorname{lmin}^{-1}$ ) is shunted through the PDA. Thus, blood that has been oxygenated already is being sent back to the lungs for oxygenation. This process is inefficient and could potentially strain the lungs and the rest of the body.

\subsection{Shear stress}

We estimate the shear stress at the rigid walls using a Fourier series decomposition of the velocity profiles $U_{n}(t)$ in Figure 9 and a Stokes boundary layer approximation. We consider the third geometry where the diameter of the cylinder is chosen to be such that the velocities and fluxes at the ventricles are consistent with the two-dimensional model. The simplification of the geometry, and in particular the sharp edges of the shunt, is such that large shear stresses are expected there.

The shear stress in the two semi-cylinders at time $t=0.16 \mathrm{~s}$ is shown in Figure 11 . The scales in the two plots are different, but are such that the shear stress is continuous on the curved surface between the planes $z=-L / 2$ and $z=L / 2$. The shear stress is different on different sides of the two plates separating the semi-cylinders. The particular time $t=0.16 \mathrm{~s}$ is during systole when the flux leaving the left ventricle is highest. Most of it leaves through the aorta, with some entering the pulmonary artery. The largest shear stress away from the shunt edges at this time $(\approx 0.41 \mathrm{~Pa})$ is lower than the generally-accepted maximum shear stress in the aorta of a healthy adult, which is in the range $(1,1.5) \mathrm{Pa}$ [37]. Although it is unreasonable to expect good quantitative agreement from our simplistic model, the shear stress distributions 
predicted by the mathematical model should be qualitatively correct and we believe can be used as a good starting point for future studies.

\subsection{Pressure}

Unlike healthy adults, where the typical pressure ranges in the aorta and pulmonary artery are approximately $(80,120) \mathrm{mmHg}$ and $(10,40) \mathrm{mmHg}$ respectively, the shunt in PDA patients enforces much smaller differences between the two vessels if similar cardiac outputs are to be encountered as in healthy adults. A PDA patient that exhibits the predicted pressure impulses in Figure 9 is unlikely to survive, let alone be asymptomatic. It is expected that the body will compensate in some way: for example by increasing the systemic and pulmonary systemic pressures $P_{p}$ and $P_{v}$. The solution was applied to a range of parameters and we believe that it is likely that the resistances in the systemic venous and pulmonary systems would decrease in PDA sufferers. This is a known control mechanism in the body: resistances are known to decrease during hypoxia.

The total cardiac output for a healthy adult at rest is $\approx 6( \pm 1.3) \operatorname{lmin}^{-1}$ [38]. However, it changes continually in response to circumstances. For example, when an adult exercises to $85 \%$ of his/her maximum heart rate, the cardiac output is on average 17.5 $( \pm 6) \operatorname{lmin}^{-1}$ [39] and Olympic athletes regularly reach cardiac outputs of $40 \operatorname{lmin}^{-1}$ [40]. Since ventricular ejection adjusts in response to different conditions, it is expected that the flux distribution outside the heart will change significantly in the presence of a PDA. However, since this mathematical model considers the same ejection volumes as in healthy adults, the cardiac output is constrained and speculations can be made mainly on the proportional distribution.

A different simulation was performed to test the hypothesis that the pressure in asymptomatic patients is similar to the pressures in healthy adults. The large differences in pressure across the shunt $(\sim 40 \mathrm{mmHg})$ were found to stimulate flow of unrealistic size from the left ventricle into the right ventricle in addition to flow from the aorta into the pulmonary artery. Most likely, neither the flow nor the pressure in asymptomatic PDA patients are similar to those in healthy adults and unusual flows perhaps with sloshing are present in the two arteries. However, although the pressures in the aorta and pulmonary artery are expected to not be as close as the predicted curves in Figure 9, they cannot be the same as in healthy adults either. The pressure difference that can be sustained across the shunt without backflow into the right ventricle is estimated to be around $10 \mathrm{mmHg}$ by the model, but this value depends on many factors such as the shunt size, the resistances and systemic pressures.

\section{Discussion}

Due to the large Reynolds and Womersley numbers and the complicated geometry of the aorta and pulmonary artery, numerical simulations are sensitive to small errors and perturbations. Our main aim was to develop the first continuum model for the quantitative and qualitative changes of blood flow through the patent ductus 
arteriosus. The main non-dimensional parameters that govern the flow are the Reynolds number $\operatorname{Re} \approx 3030$ and the Womersley number $\alpha \approx 16.5$. We have argued that these values allow us to model the flow in the core of the aorta and pulmonary artery as irrotational, and that it remains potential in the ascending aorta for most of the cardiac cycle. In addition, the geometries in the models are idealised so that a potential flow solution can be obtained analytically. Despite these idealisations, it is useful to have an analytic flow model as it gives a starting point to solving a difficult problem.

The main challenge in studying the haemodynamics in asymptomatic PDA patients is the lack of available measurements of the flow and pressure near the shunt. This led to our use of an electric circuit analogue to predict the time-dependent boundary conditions at the inlets and outlets of the geometries. The details of the model depend on the choice of the two equations that specify the ejection from the heart. The presented results assumed that the heart is a flux pump and the volume outputs are the same as in healthy adults. Many other scenarios were considered such as modelling the heart as a pressure pump, adding features such as compliance in the pulmonary and systemic circulations, resistance in the ventricles, valves at the ventricles that allow no backflow into the heart, a control mechanism for the valves and resistance across the PDA. The main observation is that during systole large volumes of blood are expected to flow backwards in the aorta towards the left ventricle due to the large differences in pressure between the two arteries. It is possible that this occurs in PDA patients since the main bifurcations at the top of the aortic arch are between the left ventricle and the shunt in most people, so this additional flow can be absorbed by the carotid and subclavian arteries.

All the cases considered were found to be sensitive to the chosen model of ventricular ejection. Future work should ideally include clinical measurements from PDA patients. In any case, the two-dimensional and three-dimensional models capture the local haemodynamics near a shunt sufficiently well provided realistic time dependence is introduced through the inlets and outlets. Many of the predicted results compare well with clinical observations. Firstly, the predicted difference in pressure between the aorta and pulmonary artery is found to be significantly less in PDA patients than in healthy humans, in agreement with measurements [9]. Secondly, the flow through the shunt can change direction during the cardiac cycle. During diastole the mathematical models show that there is backflow from the pulmonary artery into the aorta, which has also been observed [35,36]. During systole flow is expected to be shunted either towards the pulmonary systemic circulation or towards the left ventricle. The mathematical models predict that the cardiac output increases and the systemic resistances and pressure difference between the aorta and pulmonary artery decrease to compensate for the flow bypassing the oxygenation process in the lungs. This in turn suggests a mechanism for the lack of exercise tolerance and the development of cardiovascular disease in patients with PDA.

\section{References}

1. Morgan-Hughes GJ, Marshall AJ, Roobottom C (2003) Morphological assessment of patent ductus arteriosus in adults using retrospectively ECG-gated multidetector CT. Am J Roentgenol 181:749-754 
2. Friedlander E (2011) Pathology lectures. Published online at http://www.pathguy.com/sol/08569.jpg, last accessed September 2011

3. Ozmen J, Granger EK, Robinson D, White GH, Wilson M (2005) Operation for adult patent ductus arteriosus using an aortic stent-graft technique. Heart Lung Circ 14(1):54-57

4. Campbell M (1968) Natural history of persistent ductus arteriosus. Brit Heart J 30:4-13

5. Cassidy HD, Cassidy LA, Blackshear JL (2009) Incidental discovery of a patent ductus arteriosus in adults. J Am Board Fam Med 22(2):214-218

6. Wiyono SA, Witsenburg M, de Jaegere PPT, Roos-Hesselink JW (2008) Patent ductus arteriosus in adults: case report and review illustrating the spectrum of the disease. Neth Heart J 16(7/8):255-259

7. Abbott JA, Shively HH (1973) Auscultatorily silent patent ductus arteriosus: report of two cases with normal pulmonary presures. Chest 63:371-375

8. Aoyagi S, Chihara S, Fukunaga S, Mori R, Suda K (2009) Transcatheter coil embolization for patent ductus arteriosus in the elderly: report of a case and review of published work. Geriatr Gerontol Int 9(3):329-332

9. Spach MS, Serwer GA, Anderson PA, Canent RV, Levin AR (1980) Pulsatile aortopulmonary pressureflow dynamics of patent ductus arteriosus in patients with various hemodynamic states. Circulation 61:110-122

10. Becker TE, Ensing GJ, Darragh RK, Caldwell RL (1996) Doppler derivation of complete pulmonary artery pressure curves in patent ductus arteriosus. Am J Cardiol 78:1066-1069

11. Crossley KJ, Allison BJ, Polglase GR, Morley CJ, Davis PG, Hooper SB (2009) Dynamic changes in the direction of blood flow through the ductus arteriosus at birth. J Physiol 587(19):4695-4703

12. Smolich JJ, Mynard JP, Penny DJ (2011) Pulmonary trunk, ductus arteriosus and pulmonary arterial phasic blood flow interactions during systole and diastole in the fetus. J Appl Physiol 110:1362-1373

13. Hill WS, Polleri JO (1964) Elementary hydrodynamic basis of an analog of the global blood circulation. In: Pulsatile blood flow, McGraw-Hill Book Co., 407-421

14. Morrison LW, Bekey G, Brinkman CR, Assali NS (1971) Computer simulation of fetal baroreceptor function. Comput Biomed Res 3:561-574

15. Huikeshoven F, Coleman T, Jongsma H (1980) Mathematical model of the fetal cardiovascular system: the uncontrolled case. Am J Physiol 239:317-325

16. Guettouche A, Challier JC, Ito Y, Papapanayotou C, Cherruault Y, Azancot-Benistry A (1992) Mathematical modeling of the human fetal arterial blood circulation. Int J Biomed Comput 31:127-139

17. Menigault E, Berson M, Vieyres P, Lepoivre B, Pourcelot D, Pourcelot L (1998) Feto-maternal circulation: mathematical model and comparison with Doppler measurement. Eur J Ultrasound 7:129143

18. Wijngaard JPHM, Westerhof BE, Faber DJ, Ramsay MM, Westerhof N, van Gemert MJC (2006) Abnormal arterial flows by a distributed model of the fetal circulation. Am J Physiol Regul Integr Comp Physiol 291:1222-1233

19. Myers LJ, Capper WL (2002) A transmission line model of the human foetal circulatory system. Med Eng Phys 24:285-294

20. Waite L, Fine J (2007) Applied biofluid mechanics. McGraw-Hill Professional

21. Milnor WR (1989) Hemodynamics. Williams \& Wilkins

22. Caro CG, Pedley TJ, Schroter RC, Seed WA (1978) The mechanics of the circulation. Oxford Medical Publications

23. MacDonald DA (1974) Blood flow in arteries. Edward Arnold, London

24. Riley N (1998) Unsteady fully-developed flow in a curved pipe. J Eng Math 34:131-141

25. Chandran KB (1993) Flow dynamics in the human aorta. J Biomech Eng 115:611-616

26. Blyth MG, Mestel AJ (2002) The influence of geometry on inviscid decay rates in haemodynamic flows. J Fluid Mech 462:185-207

27. Zabielski L, Mestel AJ (1998) Unsteady blood flow in a helically symmetric pipe. J Fluid Mech 370:321-345

28. Batchelor GK (2009) An introduction to fluid dynamics. Cambridge University Press

29. Lagana K, Balossino R, Migliavacca F, Pennati G, Bove EL, de Leval MR, Dubini G (2004) Multiscale modeling of the cardiovascular system: application to the study of pulmonary and coronary perfusions in the univentricular circulation. J Biomech 38(2005):1129-1141

30. Fechtrup C, Kerber S, Karbenn U, Borggrefe M, Breithardt G (1993) Value of intravascular ultrasound in the diagnosis and characterization of patent ductus arteriosus in an adult patient. Eur Heart J 14:11481149

31. Gray H (1918) Anatomy of the human body. Lea \& Febiger, Twentieth edition 
32. Grossman W, Baim D (2005) Grossman's cardiac catheterization, angiography, and intervention. Lippincott Williams \& Wilkins, Seventh edition

33. Baer D (2005) Respiratory physiology - the essentials. Lippincott Williams \& Wilkins, Fourth edition

34. Reel S, Alexander I, Appling S, Arnstein P (2006) Straight A's in medical-surgical nursing: a review series. Lippincott Williams \& Wilkins, Third edition

35. Wilson N, Dickinson DF, Goldberg SJ, Scott O (1984) Pulmonary artery velocity patterns in ductus arteriosus. Br Heart J 52:462-464

36. Aziz K, Tasneem H (1990) Evaluation of pulmonary arterial pressure by Doppler colour flow mapping in patients with a ductus arteriosus. Br Heart J 63:295-299

37. Smiesko V, Johnson PC (1993) The arterial lumen is controlled by flow-related shear stress. News in Phys Sci 8:34-38

38. Levick JR (1991) An introduction to Cardiovascular Physiology. Butterworth-Heinemann Ltd., First edition

39. Rerych SK, Scholz PM, Newman GE, Sabiston DC Jr, Jones RH (1978) Cardiac Function at Rest and During Exercise in Normals and in Patients with Coronary Heart Disease: Evaluation by Radionuclide Angiocardiography. Annals of Surgery 187(5):449-458

40. McArdle WD, Katch FL, Katch VL (2009) Exercise Physiology: Nutrition, Energy, and Human Performance. Lippincott Williams \& Wilkins, Seventh edition 